\title{
Protective Clothing Based on High-temperature Thermal Radiation
}

\author{
Lu Junning ${ }^{1, ~ *}$, Mengjiang $\mathrm{Wu}^{2}$ \\ ${ }^{1}$ College of Chemical Engineering and Pharmacy, Henan University of Science and Technology, Luoyang, China \\ ${ }^{2}$ College of Water Resources and Architectural Engineering, Northwest A\&F University, Yangling, China
}

\section{Email address:}

13768583254@163.com (Lu Junning)

*Corresponding author

\section{To cite this article:}

Lu Junning, Mengjiang Wu. Protective Clothing Based on High-temperature Thermal Radiation. International Journal of Industrial and Manufacturing Systems Engineering. Vol. 4, No. 3, 2019, pp. 24-30. doi: 10.11648/j.ijimse.20190403.11

Received: October 1, 2019; Accepted: October 15, 2019; Published: October 25, 2019

\begin{abstract}
Due to the needs of today's society, there must be a particular group of people working in a high-temperature thermal radiation environment. High-temperature environments can quickly cause significant harm to the human body, while thermal protective clothing can effectively reduce the harm to the human body caused by high temperatures. We can solve this problem by building a model. Set up a multi-layer protective clothing for heat conduction formula under the temperature variation of heat conduction model of the MATLAB to map the temperature with time, $3 \mathrm{~d}$ surface figure, the variation of the temperature distribution can lead the Excel data tables, and processing the data in the table data, using MATLAB software to curve fitting, the fitting curve of time and skin temperature. At the same time, on the optimal thickness of protective clothing, can be based on the initial conditions and boundary conditions, construct the objective function, and use the improved particle swarm algorithm for solving, specific calculation will difference algorithm as local search of particle swarm optimization algorithm, the particle swarm algorithm with differential evolution algorithm is the local optimization solution as the initial population of generation of differential evolution operations, thickness of solving it is concluded that the optimal approximate solution, and it is concluded that the optimal thickness. The convection and radiation heat transfer coefficients, convection and radiation heat transfer, and skin temperature were calculated. Then, by using the extensibility of CFD simulation and the flexibility of environmental temperature setting, the human thermal radiation stress response model was embedded into the CFD simulation, to predict the real-time changes of human core temperature in a high-temperature thermal radiation environment. Combined with the core temperature threshold and exposure time, people's rescue operation time under different thermal radiation environment conditions can be reasonably scheduled and arranged to reduce the level of thermal stress, improve rescue efficiency and guarantee people's life safety. Finally, the thermal protection clothing is studied to determine the temperature distribution of each layer of thermal protective clothing. It provides a theoretical reference for the functional design of thermal protective clothing.
\end{abstract}

Keywords: Finite Difference, Particle Swarm Optimization, Optimal Numerical Solution, Thermal Radiation

\section{Introduction}

\subsection{In the Background}

Human thermal protection is an important research direction in the field of public safety. Fire fighting and rescue in fire environment may result in heat stress response due to exposure to high-temperature thermal radiation environment, high intensity of physical activity, and wearing heavy protective clothing. Of course. Thermal protective clothing is to work in high temperature or ultra-high temperature conditions for the safety protection of personnel, to avoid the heat source of injury to the human body of various protective clothing. Human skin is susceptible to temperature. When the heat flux density of human skin reaches $2.68 \mathrm{~J} / \mathrm{cm}^{2}$, that is, when the skin temperature reaches $45^{\circ} \mathrm{C}$, people will have burning pain. Second-degree burns occur when heat flux density increases to $5.02 \mathrm{~J} / \mathrm{cm}^{2}$, or when skin temperature reaches $72^{\circ} \mathrm{C}$ [1]. Therefore, people wearing thermal protective clothing can protect the skin; In the 
high-temperature environment, wearing thermal protective clothing can reduce the rate of heating of human skin and provide time for the wearer to react and escape, to avoid or reduce the harm of heat source to the human body. The heat source that causes harm to human body has a variety of forms, their properties are different, so the thermal protection performance requirements of thermal protection suits are different. At present, the primary forms of heat sources are flame (convection heat), contact heat, radiation heat, sparks and molten metal jet, high-temperature gas and steam, arc generated by high heat.

At the same time, the thermal protection performance of thermal protective clothing also has a great relationship with the heat transfer mode of the heat source. In general, heat is transferred by convection, conduction, radiation, and a combination of the three. For example, flames, hot gases, and hot steam in a heat source transfer heat by convection, contact heat, sparks, and molten metal by heat conduction and radiant heat by thermal radiation. The amount of heat transferred is usually expressed in terms of heat flux, which is the amount of heat per second per unit area in $\mathrm{kW} / \mathrm{m}^{2}$, $\mathrm{W} / \mathrm{cm}^{2}$, or $\mathrm{J} / \mathrm{cm}^{2} \cdot \mathrm{s}[2]$.

When people are fighting fire and rescue, high-temperature thermal radiation causes the body temperature to rise, and much sweating, the increase of sweat secretion can lead to dehydration and electrolyte loss.

Meanwhile, by their metabolic heat production, protective clothing and high-temperature thermal radiation triple factors (human - clothing - environment), the influence of the people in the rescue operation in body temperature, sweat, blood flow, and heart rate changes significantly, may appear pale, chest tightness, chest pain, heart palpitations and heat stress phenomenon such as syncope, serious when cardiovascular system and nervous system diseases [3].

Based on the guarantee of human life safety, to reduce high-temperature heat radiation, reduce heat stress response, it is necessary to design protective equipment. On the one hand, this protective equipment can prevent external heat radiation from harming the human body, from protecting human body parts from injury. On the other hand, it can also hinder the heat exchange between the human body and the environment, so that a large amount of metabolic heat production is accumulated in the body, thus aggravating the heat stress of the human body.

\subsection{Literature Review}

Torvi Heat transfer in thin fibrous materials under high heat flux conditions. He established the human body's thermal radiation stress response model under high-temperature thermal radiation environment, established the thermal and wet resistance clothing system for non-wearing and ordinary work clothes, and established the micro-environment-texture-external environment clothing system for protective clothing [4], which significantly improved the application scope and simulation accuracy of the model. However, there are some limitations in the whole process of the model method, which cannot solve the problem of thermal radiation. As can be seen from the human thermal radiation stress response model, it is mostly used in the working condition when the ambient temperature is less than $40^{\circ} \mathrm{C}$ [5], while the prediction accuracy is very low in the high-temperature environment. Therefore, based on Torvi et al.'s model, this paper will improve the calculation method of human physiological regulation and heat exchange to break through the limitation of poor prediction accuracy of the human thermal radiation stress response model in the high-temperature environment [6].

Pan bin. Mathematical construction of heat transfer and the inverse problem of parameter determination for thermal protective clothing and $\mathrm{Xu} \mathrm{D}$. Inverse problems of textile material design on clothing, heat-moisture comfort. We know that the inverse problem of the textile material design was first proposed by $\mathrm{Xu} \mathrm{D}$ [7]. The inverse problem is aimed at the thermal and humidity transfer of textile materials in the low-temperature environment. With human thermal and humidity comfort as the goal, the textile parameters such as thickness, porosity, and thermal conductivity of textile materials are determined optimally. They and others on the design method of the inverse problem of thermal protective clothing is relatively novel, can provide theory basis for thermal protective clothing performance improvement and scientific reference, so this article will ensure safe premise, based on the previous scholars of thermal protective clothing under the heat transfer model, combining the burn degree judgment, in order to minimize the burn degree as the goal, respectively, puts forward the thickness, porosity and thermal conductivity of the inverse problem boils down to the appropriate optimization model, and USES the numerical algorithm for solving.

Three. Fang-long zhu and Gibson P W and M. Young People such as scholars the proposed method of thermal and wet resistance clothing system and microenvironment-fabric-outer environment clothing system has been widely used in various industries. The former method can only be used to calculate the heat and moisture transfer of the garment layer when the human body is not wearing or ordinary work clothes [9]. The latter model method can be applied to calculate the heat and moisture transfer of the clothing layer when the human body is wearing protective clothing, can describe the heat exchange process between skin, fabric, and environment more accurately, and can comprehensively analyze the influence of material properties on the heat and moisture exchange [10].

Four. Ahmed Ghazy. Moreover, Bergstrom D. put forward Numerical simulation of heat transfer in firefighters' protective clothing with multiple air gaps during flash fire exposure. The principles and methods of their models are similar. According to typical human thermal physiology and heat exchange parameters, such as core temperature, skin temperature, and evaporative heat exchange, they verify the validity of the established human thermal response model and apply it to the evaluation of human thermal comfort and thermal stress [11].

Five. Zhang wen sheng, she put forward finite difference 
method of the partial differential equation based on scientific calculation. Higher education press. This method improves the precision of the numerical solution but increases the computation. Therefore, in this paper, based on the principle of finite difference method, we improved the accuracy of the whole model method [12].

Six. Pan Feng, He put forward particle swarm optimization algorithm and multi-objective optimization. His model is one of the most advanced methods in the world, which can have excellent compatibility for any thermal radiation problem, and the accuracy of the solution is the most accurate, and the conclusion obtained is the most accurate. Widely cited by many scholars [13].

Seven. Zhang Wei yuan. He put forward Clothing comfort and function. Beijing: China textile press and Liu Liying. Numerical simulation of human microclimate heat and humidity transfer and establishment of human thermal comfort sensation model. The model method, compared with the traditional human body thermal radiation thermal model is only exciting environment, and the body wear protective clothing when the characteristics of the simulation precision is reduced, its scope of application and simulation precision has the considerable enhancement, the body can well realize the environment of high temperature - clothing - environment of heat transfer and the calculation of the thermal physical parameters, the model values with the experimental measurements are also very consistent, meet a fireman fire fighting and rescue people body heat comfort and thermal stress evaluation of demand.

Eight. Qiu man, Wu Jian-min, chang Shao-Yong, song DE. They put forward a study on the regulation mechanism of human sweating in different activity intensity under different ambient temperatures. Chinese Journal of applied physiology. In the human body thermal radiation thermal modeling, will take the initiative to vasodilation and vasoconstriction, trembling and sweating control coefficient can't reflect the individual difference, so in the future research, we can be obtained through the human body thermal physiological experiment and heat transfer under the environment of high-temperature thermal regulation parameters of different groups. Based on the model method, this paper also focuses on the heat exchange between the human body, clothing, and environment and the human thermal response of firefighters in the high-temperature environment. The evaporation and heat exchange of sweat in the high-temperature environment is crucial to the regulation of normal body functions. In fact, there are many factors that cause sweating, such as nervous sweating (such as people in a tense state), taste sweating, and temperature sweating. The nervous sweating caused by the complexity and danger of the fire scene. However, the influence of psychology on human thermal regulation has not been considered in this paper, and studies on the influence of psychology on thermal regulation behavior have never been reported in the existing literature. Therefore, in order to make the model more realistic to simulate the actual thermal physiological response of firefighters in the fire site, it is necessary to study the psychological influence on the thermal regulation behavior.

\subsection{Research Purpose and Significance}

Human thermal radiation response has become one of the most active research directions in today's society [14], widely used in aerospace, ergonomics, biomedicine, and public safety and other fields. Due to the complexity of the mission environment and the high requirements on the design of the spacecraft environment module [15], spacesuit, and human thermal comfort, the research on human thermal radiation response has been greatly promoted in the field of human-crewed spaceflight [16]. Based on this research, human thermal radiation response research can achieve human comfort research in architectural design, HVAC and other fields. In modern biomedical engineering, the human body temperature field can be obtained and described for medical diagnosis. In the field of human ergonomics and thermal protective equipment, the design and evaluation of thermal protective equipment can be carried out, and in the field of public safety, it can be used to guide emergency rescue, personnel thermal comfort and thermal stress evaluation, research and development and testing of protective equipment.

Because the human body can produce heat through metabolism by a chemical reaction between food and oxygen and exchange heat with the external environment through convection [21], radiation, evaporation, and conduction. In order to evaluate the physiological parameters of the human body in the environment of high-temperature thermal radiation more scientifically and reasonably, reduce the level of heat stress, improve rescue efficiency, and guarantee life safety. Therefore, this paper will establish a thermal design model of thermal protective clothing under high temperature environment, and make an in-depth understanding of the internal heat transfer rules of thermal protective clothing, which will provide a practical and reliable theoretical basis for high-temperature operators to avoid skin burns in high temperature, thus providing scientific guidance for the design of thermal protective clothing.

With the urgent need for human life safety and protective equipment development in the field of human thermal radiation protection for public safety, the study on human thermal radiation stress response in the high-temperature environment is of considerable significance $[17,18]$.

First, the physiological parameters of human thermal radiation in the high-temperature environment were predicted, and thermal comfort and thermal stress were evaluated.

The comprehensive heat transfer, physiology, materials science, human ergonomics and numerical calculation, multidisciplinary, and through these disciplines knowledge to build up human body thermal stress model to predict the human body thermal physical parameters, such as skin and core temperature, sweat, blood flow and heart rate, etc., will calculate the physiological parameter values can be used for thermal comfort and thermal stress evaluation.

Secondly, it provides technical support for the testing of thermal protective equipment under the human body's 
thermal radiation environment and for research and development.

For example, we can couple the warm body dummy with the established human thermal response model, to further realize the intelligence of the dummy. Then, the physiological parameters obtained from the model simulation are used to control the warm body dummy, so that the dummy can have the physiological regulation function and can "feel" the change of the thermal radiation environment and make for dynamic adjustment. By using the coupling system of dummy and model to obtain human physiological parameters, the comparison of human physiological parameters under different dressing conditions can provide support for testing and research and development of protective equipment.

Third, through countermeasures to guide fire emergency rescue.

In high-temperature thermal radiation, the environment can cause human burns and even endanger the safety of life. Therefore, we can use the extensibility of CFD simulation and the flexibility of environmental temperature setting to embed the human thermal radiation stress response model into the CFD simulation, to predict the real-time changes of human core temperature in the high-temperature thermal radiation environment. Combined with the core temperature threshold and exposure time, people's rescue operation time under different thermal radiation environment conditions can be reasonably scheduled and arranged to reduce the level of thermal stress, improve rescue efficiency and guarantee people's life safety.

As is known to all, we only need to consider the two primary forms of heat transfer of thermal protective clothing in the high-temperature thermal radiation environment and analyze the heat conduction in the high-temperature thermal radiation environment and the effect of heat radiation on the heat transfer process.

\subsection{Heat Radiation Two Forms of Heat Transfer in a High-temperature Environment}

Thermal radiation

Radiant heat is one of the significant forms of heat transfer that can cause injury to a victim, just as eighty percent of the energy produced by burning a flame is produced by thermal radiation.

Radiation is the way that an object transmits energy through electromagnetic waves, and the electromagnetic wave radiation generated due to heat is called thermal radiation.

Thermal radiation, the phenomenon of electromagnetic waves radiated by an object due to its temperature. One of the three ways that heat is transferred. Any object whose temperature is higher than absolute zero produces heat radiation. The higher the temperature, the greater the total energy emitted, and the more short-wave components there are. The spectrum of thermal radiation is continuous, and the wavelength coverage range can theoretically be from 0 to infinity. Because electromagnetic waves travel without any medium, thermal radiation is the only way to transfer heat in a vacuum.

The electromagnetic radiation caused by heat is called thermal radiation. It is excited by the change of motion of the microscopic particles inside the object. The energy is divided into infrared, visible, and ultraviolet. The thermal effect of infrared rays on the human body is significant.

Characteristics of thermal radiation:

1. Any object, as long as its temperature is higher than $0 \mathrm{~K}$, will continuously emit thermal radiation to the surrounding space.

2. Can be spread in vacuum and air.

3. Accompanied by the transformation of energy forms.

4. Strong directivity.

5. Radiant energy is related to temperature and wavelength.

6. The emission of radiation depends on the temperature to the fourth power.

Heat conduction

In the practical application of thermal protective clothing, this paper only considers two forms of heat transfer. The energy transfer caused by temperature difference is heat transfer [19]. At any time, as long as there is a temperature difference between a particular medium or two media, heat transfer will occur. The phenomenon of heat conduction does not involve macroscopic migration of matter. Heat from high temperature to the low-temperature part of the heat transfer, heat transfer is also called thermal diffusion, Fourier law is to describe the fundamental laws of heat conduction in the reference materials are described in detail in this paper, the heat transfer rate ability of thermal conductivity properties of the material thermal conductivity is different, its size is profoundly affected by the temperature, such as thermal protective clothing materials Nomex, under the condition of high-temperature thermal conductivity is changing with temperature have essential effects on the heat transfer process [20].

Heat conduction is no macroscopic movement at the time of the heat transfer phenomena in the medium, its all can happen in solid, liquid and gas, but strictly speaking, only is the pure heat conduction in solids, and fluid even in static state, which will be due to the temperature gradient caused by the natural convection resulting from the density difference, therefore, the heat convection and heat transfer in the fluid at the same time.

\section{The Development Process of Thermal Protective Clothing Research}

In recent years, the study of thermal protective clothing heat transfer model, the internal and external scholars have different degree of progress, in the existing model according to the thermal resistant clothing is adopts single layer or multi-layer materials, and is divided into the single-layer and multilayer model, so-called single model of hot, it is only the shell, the domestic and foreign scholars mainly research Gui 
its external flame radiation heat, the physics properties of fabric, the fabric and the thickness of air layer between the skin effect the performance of the protective clothing thermal Gibson M [4] first proposed: Heat and mass transfer model of single-layer porous media under high-temperature condition, but this model does not adequately consider the influence of thermal radiation in the fabric layer. Therefore, in order to improve the model, Tend $\mathrm{M}$ redefines the heat transfer model of thermal protective clothing shell material under the condition of long-time exposure to intense radiation and low radiation. Considering that the thickness of air layer has a particular impact on the characteristics of thermal protective clothing, Torvi [1] establishes a model to calculate the internal heat transfer and skin injury time during the cooling process based on considering the influence of the thickness of air layer on the thermal performance of protective clothing. In the cylindrical coordinate system, zhu fang long [3] studies the heat transfer process under different curved surfaces. Experimental device based on ASTM D 4108 [5], forecast Sawcyn [7] developed protective clothing and heat transfer model of the air layer, on the basis of predecessors' study, Ghazy system to hot clothing - air - proof skin of each link is very detailed and profound research: heat conduction and heat by empirical formula of single layer fabric, from constants to variable, is more aptly fitting the condition of high-temperature heat transfer process. A simple and accurate air layer model is proposed. The heat transfer model of single layer fabric in motion is established. Based on the single-layer model, many scholars have studied the multi-layer model of heat and moisture transfer of thermal protective clothing. Mell [8] proposed a heat transfer model between layers. Because the phase change material has certain influence on the thermal protection effect. Mercer and Ahmed Elgafy [9] established a multilayer dynamic heat transfer model containing phase change materials. Considering the effect of moisture on the thermal protection effect, Lawson further developed a heat and moisture transfer model for multi-layer fabrics. Considering the effect of air layer on the performance of thermal protective clothing, Ghazy established a multilayer fabric heat transfer model with multiple air layers. As for the design of multi-layer thermal protective clothing, Fan established the heat and humidity transfer model of textile materials at low temperatures. In order to ensure the requirement of heat preservation, he proposed the inverse problem of porosity determination aiming at the minimum thermal damage. At the same time, based on professor fan jintu's model, $\mathrm{Xu}$ [11] proposed an inverse problem of textile material design based on clothing comfort. For single-layer fabrics, IPTMD includes the inverse problem of fabric thickness determining, fabric porosity determining, fabric thermal conductivity determining and so on. For multilayer fabrics, IPTMD is a multilayer multiparameter inverse problem.

In other research directions, some scholars Song $\mathrm{G}$ et al. studied the heat and moisture transfer model of thermal protective clothing under instantaneous exposure flame, obtained the distribution of temperature and moisture in the fabric and air layer through model and experiment, and evaluated thermal damage by combining skin model. The results clearly show that the thickness of the air layer has a significant effect on the performance of thermal protective clothing.

\subsection{Based on the Finite Difference Method}

Firstly, the finite difference method is used to solve the nonlinear parabolic square, which is constructed by a multilayer heat protection suit, air layer and skin Cheng group The main idea is to replace the continuous definite solution region with a grid composed of a finite number of discrete points, which is called the node of the grid. The functions of continuous variables in the continuous domain are approximated by the functions of discrete variables defined on the grid nodes. The difference quotient is used to approximate wechat business in the original equation and the definite solution condition, and the integral sum is used to approximate the integral, so the definite solution condition and the original differential equation are converted into a finite difference system of equations, we only need to solve the equations to get the numerical solution of the original problem at discrete points.

In this choice by discrete method to solve the problem, the purpose is to better convenient for data in the grid computing, using MATLAB to calculate, by programming program for numerical solution can get the final data, the desires of each point is obtained within the prescribed period of time tend to be the most considerable stable temperature, based on this, it can be made temperature distribution and the three dimensional temperature variation.

\subsection{Hybrid POS Algorithm Based on Fde}

For the most favorable problem of thermal protection, the ambient temperature can be converted to $65^{\circ} \mathrm{C}$ and the thickness of IV layer is $5.5 \mathrm{~mm}$, and the constraint conditions can be increased. Then, the improved particle swarm optimization algorithm is used to approximate the solution of the equation. By using the difference method as a sub-algorithm of PSO for local search, and using the differential evolution algorithm as an initial population to perform a series of generation differential evolution operations, the local optimal solution can be better obtained. It makes the particle swarm optimization (PSO) with poor local search ability more convergent and fully differential evolution algorithm. The improved particle swarm optimization (PSO) algorithm has strong local searchability and is more suitable for excellent search.

\subsection{This Hybrid POS Algorithm Steps}

Step 1: randomly generate the initial population of $\mathrm{N}$ in search space s $X_{(\mathrm{i} 0)}(\mathrm{i}=1,2, \cdots, \mathrm{N})$, initialize the velocity of each particle initialized $V_{(i 0)}=(i=1,2, \cdots, N$, initialize the position of each particle, and compare 
$P_{g} \operatorname{and}_{(i 0)}(i=1,2, \cdots, \mathrm{N})$, one by one according to plan 2 .

The tolerance is defined, and the maximum iteration of the population evolution algebra is set, and the current iteration number $\mathrm{t}=0$ is set.

Step 2: if t $>$ iteration, stop, and output $P_{g}$. Otherwise, go to step 3 .

Step 3: calculate the velocity $V_{1(\mathrm{t}+1)}$ and position $X_{1(\mathrm{t}+1)}$ of each particle according to formula, compare $P_{1(t)}$ and $V_{1(t+1)}$ according to plan 2, and update $P_{1(t)}$.

Step 4: set the differential evolution algebra $\mathrm{k}$, set the current differential evolution algebra $\mathrm{d}=0$, and locally optimize the solution of each particle

A performs the following operations:

Step 4.1: perform mutation operation on the local optimal solution to get sub-individuals, where $P_{1(t)}$ are two random integers in $V 1(t)$.

Step 4.2 conduct hybridization between parent and child to produce individual $u_{1(t 0)}$, if $\operatorname{randi}_{i j} \leq C R$, otherwise $\mathrm{j}=1.2$.

Step 4.3: where $P_{1(t)}=p(t)+\operatorname{rand} 2\left(P_{1(t)}-P_{2(t)}\right)$. rand 2 is a random number in the interval $(0,1)$ subject to a uniform distribution.

Step 4.4: compare $P_{1(\mathrm{t})}$ and $P_{\mathrm{i}(\mathrm{t})}$ one by one according to the scheme, and mark the superior as $P_{1(\mathrm{t}+1)}$. According to the scheme, $P_{g}$ it will be compared with $P_{1(\mathrm{t}+1)}, \mathrm{P}_{2(t+1)} \cdots, P_{N(t+1)}$ one by one and $P_{g}$ will be replaced by the better one.

Step 5: $\mathrm{d}=\mathrm{d}+1$ if $\mathrm{d}<\mathrm{K}$ moves to step 4.1 .

Step 6: $\mathrm{t}=\mathrm{t}+1$, go to step 2 .

According to the above steps of the HPSO algorithm [12], the programming code of MATLAB is established, and then the obtained equation and known quantity are substituted into the solution to obtain the optimal thickness range of thermal protective clothing.

\section{Conclusion and Recommendation}

Heat protection of the human body is an important research direction of human life safety. Heat stress and burn caused by high-temperature environments are essential factors threatening the life safety of firefighters in the process of fire fighting and rescue. In this paper, human thermal response in a high temperature environment is studied. Based on the knowledge of physiology, heat transfer, material science and numerical analysis, finite difference method and particle swarm optimization algorithm are adopted. Through detailed qualitative analysis, as well as graphics, to clearly show the relationship between the various variables of this type of question.

For the high-temperature environment, the heat transfer model of single-layer thermal protective clothing is fully considered, and the heat conductivity and heat capacity per unit volume of the fabric and air change with the temperature at high temperature, which has a significant influence on the heat transfer process of the model.

Based on the results of the model, we can propose several questions worthy of discussion.

1. Further, improve the heat transfer model of thermal protective clothing -- air layer -- skin system

The thermal protective clothing - air layer - skin system established in this paper still has many aspects of being improved. For thermal protective clothing, this paper presents the heat transfer model of multi-layer thermal protective clothing, without considering the wet transfer. It often has an essential influence under high temperature and cannot be ignored. For the air layer, a simplified model is adopted to calculate the air layer, and the heat transfer model of the air layer model can be further improved. The heat transfer model in this paper does not look at the influence of moisture transfer, and the influence of moisture transfer on thermal protective clothing can be further considered.

2. Heat and moisture transfer of multi-layer thermal protective clothing

In this paper, we give the heat transfer equation of single-layer thermal protective clothing. However, many thermal protective clothing fabrics are multi-layers. For example, in multi-layer thermal protective clothing of fire protection clothing, there is an air layer between layers, which has an essential influence on heat transfer. There is bound to be water vapor generation (including sweat and water vapor in the environment). However, moisture transfer is not considered in this paper, and the thermo-humidity coupling model can be further considered in later studies.

All in all. We established the differential equation model of time and human surface temperature, and through qualitative analysis of it and curve fitting, we clearly showed the relationship between various variables in the problem. At the same time, through different improved particle swarm optimization algorithms, the optimal approximate solution of the programming problem can be obtained very well, but the algorithm has higher iteration times and massive computation, and the algorithm is relatively stable.

In view of the thermal radiation in high temperature environment, consider single heat transfer model of thermal protective clothing, we analyzed the fabric under high temperature and thermal radiation and thermal conductivity and heat capacity per unit volume of air is along with the temperature change, and the change to the model of heat transfer process is a significant influence. At the same time we are on the problem model that is simulated and through the graphical analysis. At the same time, in order to better illustrate the rationality of the proposed model study, the numerical results in this paper are compared with the numerical results of the existing experimental demonstration of Torvi, and the numerical results are proved to be reasonable by setting the same parameter conditions as the error of the numerical results. 


\section{References}

[1] Torvi Heat transfer in thin fibrous materials under high heat flux conditions [D]. Edmonton: University of A lberta, 1997: $1-134$.

[2] Pan bin. Mathematical construction of heat transfer and inverse problem of parameter determination for thermal protective clothing [D]. Zhejiang university of science and technology, 2007. (in Chinese)

[3] Fang-long zhu. Thermal protection function of clothing, Beijing: China miscellaneous weaving press, 2005.10. (in Chinese).

[4] Gibson P W. Multiphase heat and mass transfer through hygroscopic porous media with applications to clothing materials [J]. Fiber, 1996, 53 (5); 183-194.

[5] ASTM D 4108-87. Standard test method for thermal proformance of materials and clothing by open-flame method [S]. American Society for Testing $M$ aterials, West Conshohocken, PA, 1987.

[6] M. Young, The Technical Writer's Handbook. Mill Valley, CA: University Science, 198.

[7] Saweyn C M J. Torvi D A. Improving beat transfer models of air gaps in bench top tests of thermal protective fabrics [J]. Textile Research Journal, 2009, 79 (79): 632-644.

[8] Mell W E, L awson J R. A Heat transfer model for firefighters' protective clothing [J]. Fire Technology, 2000, 36 (1): 39-68.

[9] Ahmed Ghazy. Bergstrom D. Numerical simulation of heat transfer in firefighters' protective clothing with multiple air gaps during flash fire exposure [J]. Numerical Heat Transfer, 2012, 61 (8): 569-593.

[10] Du N, Fan J, Wu H, et al. Optimal porosity distribution of fibrous insulation [J]. International Journal of Heat and Mass Transfer, 2009, 52 (19-20): 4350-4357.

[11] $\mathrm{Xu} \mathrm{D}$. Inverse problems of textile materilal design on clothing, heat-moisturecomfort [J]. Applicable Analyais, 2014, 93 (11): 2426-2439.

[12] Zhang wen sheng, finite difference method of partial differential equation based on scientific calculation. Higher education press, 2006 (in Chinese).

[13] Pan feng, particle swarm optimization algorithm and multi-objective optimization, 2013 (in Chinese).

[14] Zhang wei yuan. Clothing comfort and function. Beijing: China textile press, 2011 (in Chinese).

[15] Wang zhao jun, hou Juan, kang cheng zu, ning haoran. Experimental study on human thermal response in asymmetric radiant heat environment. Hvac, 2015, (6): 59-63.

[16] Qiu man, wu jian-min, chang shao-yong, song DE. Study on the regulation mechanism of human sweating in different activity intensity under different ambient temperature. Chinese journal of applied physiology, 2005, 21 (1): 90-94.

[17] Stapleton J M, Wright H E, Hardcastle S G, et al. Body heat storage during intermittent work in hot-dry and warm-wet environments. Applied Physiology Nutrition and Metabolism, 2012, 37 (5): 840-849.

[18] Z. Zhao, J. Wang and Y. Liu, "User Electricity Behavior Analysis Based on K-Means Plus Clustering Algorithm," 2017 International Conference on Computer Technology, Electronics and Communication (ICCTEC), Dalian, China, 2017, pp. 484-487. doi: 10.1109/ICCTEC.2017.00111.

[19] Vesely M, Zeiler W. Personalized conditioning and its impact on thermal comfort and energyperformance - a review. Renewable and Sustainable Energy Reviews, 2014, 34 (3): 401-408.

[20] Yu chang ming. Heat conduction and numerical analysis [M]. Tsinghua university press, 1981 (in Chinese).

[21] Liu liying. Numerical simulation of human microclimate heat and humidity transfer and establishment of human thermal comfort sensation model [D]. Dong hua university, 2002.

[22] Zhang Yanjun, Yang Xiaodong, Liu Yi, Zheng Dayuan, Bi Shujun. Research on the Frame of Intelligent Inspection Platform Based on Spatio-temporal Data. Computer \& Digital Engineering [J], 2019, 47 (03): 616-619+637.

[23] Yang jie, weng wen guo. Prediction of physiological parameters based on high temperature human thermal response model. Journal of tsing hua university (natural science edition), 2014, 54 (11): 1422-1427. 